\title{
Time resolved excitonic coherent polarization dynamics in GaAs multiple quantum wells
}

\author{
Bipul Pa: and A. S. Vengurlekar \\ Tata Institute of Fundamental Research, Mumbai 400005, India
}

(Dated: November 8, 2018)

\begin{abstract}
We investigate pump induced modulation in the temporal evolution of the excitonic coherent polarization generated by a probe pulse in GaAs multiple quantum wells. For this, we perform fs time resolved pump-probe reflectivity measurements using frequency upconversion at both positive and negative pump-probe delays. A contribution arising from interference of excitonic linear and nonlinear polarization, which is usually not considered in describing pump-probe experiments, is required to understand the results.

PACS numbers: 71.35.-y, 78.67.De, 78.47.+p, 82.53.Mj
\end{abstract}

Linear and nonlinear optical response of excitonic resonances in GaAs like semiconductor multiple quantum wells (MQW) to excitation by ultrashort laser pulses and the behavior of the primary and secondary radiation emitted by the excited macroscopic polarization has been of much interest in recent years. Measurement of time resolved emission in the reflection ${ }^{1.2 .3}$ and transmission ${ }^{4.5}$ directions as a result of resonant excitation of excitons in quantum wells (QW) by an ultrashort laser pulse showed a time-profile significantly different from that of the incident pulse as a result of coherent emission from the excitonic polarization surviving up to several ps. A modification in the temporal evolution of the coherent emission in the probe reflection and transmission direction occurs in pump-probe differential (PPD) measurements. This is caused by linear and nonlinear interactions among the populations and polarizations, excited in the MQW by the probe and the pump pulses, and the propagating electric fields. An interesting manifestation of the nonlinear optical effects in the pump-probe differential transmission ${ }^{6.7 .8 .9}$ (PPDT) and reflection ${ }^{10.11}$ (PPDR) is the transient oscillations observed in spectrally resolved (SR) measurements for negative ( - ve) pump-probe delay $(\tau)$. (Here, - ve delay corresponds to the situation when the probe pulse precedes the pump pulse.) As the PPD measurements entail detection of intensity either of the spectrally or time resolved signals, and as these intensities are not simply Fourier related, important phase related effects are not the same in the two measurements. Therefore, additional insights into the modification of the emission in the probe reflection by a pump pulse may be obtained from time domain measurements. However, to our knowledge, such measurements have not been reported so far.

The purpose of this paper is two-fold. First, we present our experimental investigations of the time resolved PPDR from excitons in GaAs MQWs performed using the frequency upconversion technique. We find that the modulation in the dynamics of probe induced excitonic coherence by a pump pulse at low intensities occurs with a rise time of a few ps. However, the rise becomes faster as the pump intensity increases. No signal having the pump pulse polarization is emitted along the probe reflection direction for pump-probe cross-linear polarization. A PPDR signal is observed for - ve delay in both time resolved (TR) and time integrated (TI) measurements. The other main objective of this paper is to point out that the theoretical expressions usually employed to explain the PPD measurements ${ }^{12.13}$ are inadequate in explaining the TR- and TI-PPDR measurements. Traditionally, the PPD signal is expressed as an interference of the probe electric field with the excitonic nonlinear polarization. This was able to explain the SR-PPDT results 8.14 .15 in the regime of the third order nonlinear optical susceptibility. One prediction of this description is that no signal in TR-PPD measurements can occur for $\tau<0$. This theory allows nonzero signal only for $\tau>0$ and that too at the time of arrival of the probe pulse in a narrow time domain limited by the probe pulse width. Furthermore, the model also predicts that the TI-PPD (or equivalently, spectrally integrated (SI) PPD) signal at - ve delay should vanish. However, all these predictions are contradictory to our experimental results. To understand the origin and nature of the signals observed in TR- and TI-PPDR measurements, we reexamine the theory for calculating the PPD signals. We show that an additional nonlinear optical contribution arising from interference of excitonic linear and nonlinear polarization, which is usually ignored in the literature, has to be invoked to explain our results.

Our experiments are performed at $8 \mathrm{~K}$ on $17.5 \mathrm{~nm}$ thick GaAs multiple-QWs, 20 in number, separated by $15 \mathrm{~nm}$ $\mathrm{Al}_{0.33} \mathrm{Ga}_{0.67} \mathrm{As}$ barriers, with a $330 \mathrm{~nm}$ AlGaAs layer on top. Continuous wave photoluminescence spectra measured at $8 \mathrm{~K}$ reveal heavy hole (hh) exciton emission at $1.5305 \mathrm{eV}$, with a spectral full width at half maximum (FWHM) $0.8 \mathrm{meV}$. Ti-Sapphire laser pulses of width of $180 \mathrm{fs}$ (spectral FWHM of $10 \mathrm{meV}$ ) and a repetition rate of $82 \mathrm{MHz}$ are used to resonantly excite the hh excitons. However, as the hh and light hole (lh) exciton energies are separated by $5.7 \mathrm{meV}$, coherent excitation of lh excitons also occurs. Dependence of TI- and TR-PPDR signal on the pump-probe delay is measured using the experimental arrangement shown schematically in Fig. 1(a). The TR-PPDR signal as well as the time profile of the reflected probe pulse in absence of a pump pulse are measured using type-I frequency upconversion in a $\mathrm{LiIO}_{3}$ crystal on which the reflected probe pulse and a reference pulse are cofocussed. The pump, probe and reference pulses are all derived from the same laser beam. Figure 1(b) schematically shows the pulse 


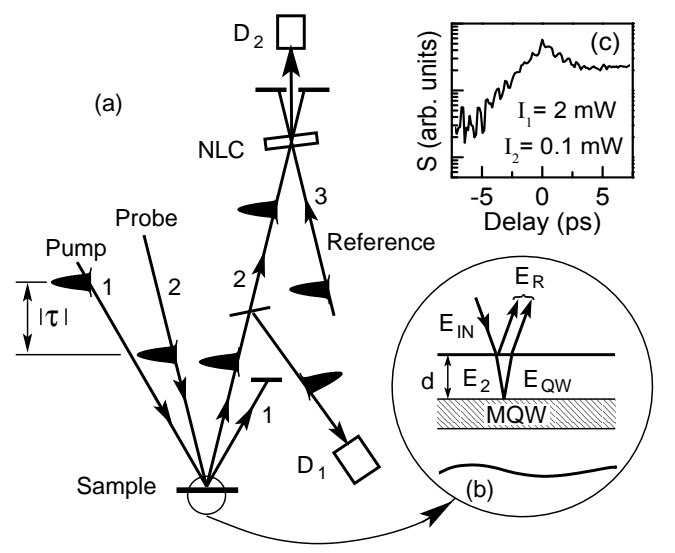

FIG. 1: (a) Schematics of the experimental arrangements. NLC = nonlinear crystal, D = photomultiplier tube detector. (b) The propagation directions for the incident $\left(E_{I N}\right)$ and reflected $\left(E_{R}\right)$ probe pulse are shown, together with a schematic picture of the sample having the MQW and a top layer (of thickness $d$ ). $E_{2}$ is the incident probe electric field on the QWs and $E_{Q W}$ is the field emitted from the QWs. (c) The magnitude of the time (and spectrally) integrated PPDR signal as a function of pump-probe delay is plotted on a semilogarithmic scale. $I_{1}\left(I_{2}\right)$ is the average intensity of the pump (probe) pulse.

propagation directions for the incident and reflected probe beam in the MQWs.

Delay dependence of the magnitude of the time (and spectrally) integrated PPDR signal (which is actually -ve) is plotted in Fig. 团(c). Note that the TI-PPDR signal does not vanish at - ve delay up to about -6 ps. The signal decay for $\tau<0$ is nearly exponential with a time constant of about 2 ps. For large positive ( + ve) delay, the signal decay is slow and is presumably governed by the exciton life time (full data is not shown). At small +ve delay, an additional fast decaying component is seen. The oscillations seen in the data at -ve and small + ve delay are the signature of quantum beats due to coexcitation of hh and lh excitons.

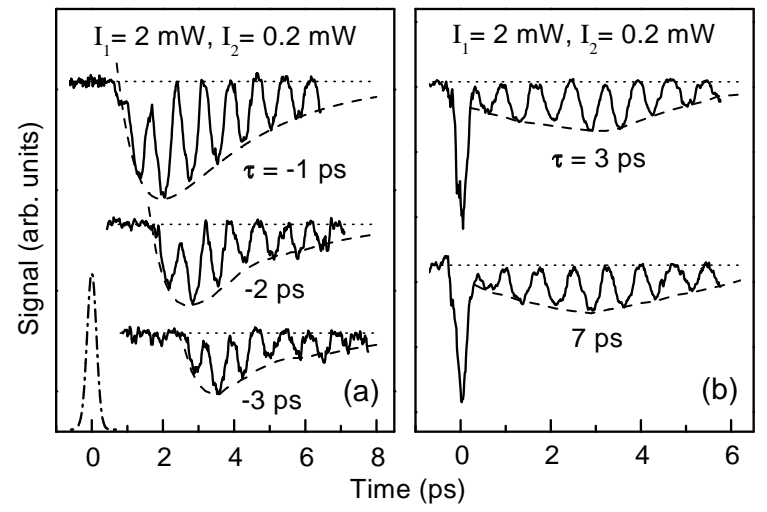

FIG. 2: Time evolution of the TR-PPDR signal (solid lines) at a few - ve (a) and +ve (b) delays are shown on linear scales. Here pump (probe) intensity $I_{1}\left(I_{2}\right)=2(0.2) \mathrm{mW}$. The signal is - ve and the data for different $\tau$ are shifted along the vertical axis, with the zero of the signal shown as dotted lines. The dashed lines are shown as the envelope of the signal to guide the eye. The cross-correlation trace for the probe pulse is plotted in (a) (dashed-dotted line).

The TR-PPDR measurements are shown in Figs. 2 and 3 for both - ve and + ve $\tau$. Here, we take the probe pulse to arrive at $t=0$ and the pump pulse at $t=-\tau$, so that the pump pulse arrives after (before) the probe pulse when $\tau<0(\tau>0)$. The average pump (probe) intensity $I_{1}\left(I_{2}\right)$ is $2(0.2) \mathrm{mW}$ in Fig. [2] For the data in Fig. [3] $I_{1}$ is varied, keeping $I_{2}$ fixed at $0.3 \mathrm{~mW}$. The data shows quantum beat oscillations (Figs. 2] and 3) with a period of about $0.75 \mathrm{ps}$, corresponding closely with the hh-lh exciton energy separation of $5.7 \mathrm{meV}$. Also, shown in Fig. 2(a) (dash-dotted line) is the cross-correlation trace of the laser pulse, giving the time resolution of the upconversion measurement of about 250 fs. Note that the TR-PPDR signal in Figs. 2 and 3 is - ve at all $t$. This means that the coherent polarization induced by the probe pulse in the MQWs is reduced due to the influence of the pump pulse for both - ve and +ve delay. We show later that such a reduction may be explained by invoking effects like phase space filling and excitation induced dephasing within the framework of optical Bloch equations. (However, local field or exciton energy shift have no role in this.) 
Consider first the case of $\tau<0$. The emission along the probe reflection direction following excitation by the probe pulse may survive so long as the exciton polarization radiates coherently. A pump pulse incident during this process modifies the subsequent emission. Figure2 (a) shows the time evolution of the PPDR signal measured at three - ve delays. As expected from causality, the onset of the TR-PPDR signal correlates with the arrival of the pump pulse, delayed with respect to the probe pulse. No signal is observed when $\tau$ exceeds about 8 ps as the probe pulse induced polarization dephases within this time. At low intensities, the signal shows a rise time larger than the laser pulse width. After reaching the peak, it decays approximately exponentially with a time constant of about 2.5 ps. Figure 3(a) plots the TR-PPDR signal measured at three values of average pump intensity $I_{1}$ at $\tau=-1$ ps. While the decay rate of the signal appears to be rather insensitive to $I_{1}$, a reduction in rise time with increased $I_{1}$ is clearly seen.

The observation of a nonzero TR-PPDR signal even when the pump pulse is incident on the sample well after the probe pulse has passed may be compared with a similar situation noted previously in the case of four wave mixing (FWM) measurements 16.17 . However, within the regime of the third order nonlinear optical susceptibility, the nonlinear optical polarization relevant to the PPD is not the same as that in FWM. For example, transient oscillations are seen in SR-PPD measurements at - ve delays, but not in SR-FWM. The exact origin of the signals at - ve $\tau$ is different in the two cases. In the FWM case, the polarization induced by the pulse along $\mathbf{k}_{2}$ gets diffracted along $2 \mathbf{k}_{2}-\mathbf{k}_{1}$ after the other excitation pulse arrives along $\mathbf{k}_{1}$. This is a result of many body Coulomb interactions ${ }^{17.18 .19}$ (such as local field, biexcitons, excitation induced dephasing, for example). On the other hand, the TR-PPDR signal at $\tau<0$ is in principle possible within the third order nonlinear optical regime even without introducing the many body Coulomb interactions, as shown presently.

It is sometime stated that the SR-PPD signal observed for $\tau<0$ is a result of pump pulse being scattered in the probe reflection/transmission direction from a grating created by the probe induced exciton polarization and the pump pulse ${ }^{8.13 .15}$. If a transfer of energy from the pump beam to the probe reflection direction occurs, this may give rise to a +ve TR-PPDR signal, contrary to the data of Figs. 22(a) and 31a). We further investigate this point by performing the TR-PPDR measurements for the pump polarization parallel and perpendicular to the probe (and the reference pulse) polarization. We find that the TR-PPDR signal which is measured using type-I upconversion is identical for the two cases of pump polarization. This confirms that the emission in the probe reflection direction always has the polarization of the probe pulse ${ }^{20}$.

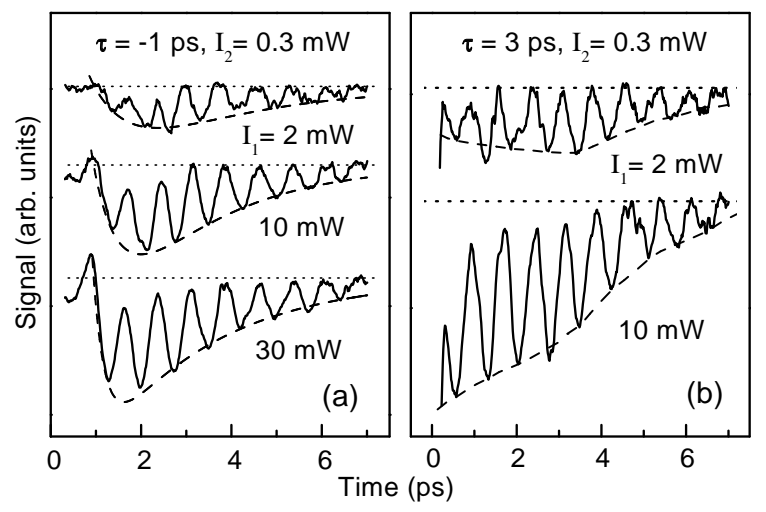

FIG. 3: Time evolution of the TR-PPDR signal (solid lines) at a few pump intensities $\left(I_{1}\right)$ at $\tau=-1$ ps (a) and 3 ps (b), with $I_{2}=0.3 \mathrm{~mW}$. The signal is - ve and the data for different $\tau$ are shifted along the vertical axis (linear scale), with the zero of the signal shown as dotted lines. The dashed lines are shown as the envelope of the signal to guide the eye.

Figure 2(b) displays the modification caused by a pump pulse in the time evolution of radiation from the excitonic polarization induced in the MQWs by a probe pulse incident after a +ve delay $(\tau>0)$. Unlike $\tau<0$, the TR-PPDR signal in this case has a large contribution at $t=0$, and then a tail for $t>0$. The signal at $t=0$ is essentially caused by the interference of the probe electric field with the excitonic nonlinear polarization induced by the pump and probe pulses. The signal in the tail part shows a rise and then a decay with $t$. The rise for $\tau>0$ [Fig. 22(b)] is slower compared to the $\tau<0$ case seen in Fig. 2(a). In fact, the overall shape of the $t$ dependence is different in the two cases [Figs. 2(a) and (b)]. Also, the TR-PPDR signal shows only a small dependence on the value of $\tau$ when $\tau$ is +ve. (We verify this by varying $\tau$ from 1 to $30 \mathrm{ps}$.) The pump pulse excites its own coherent exciton polarization which turns into incoherent excitons after several ps. The rather small sensitivity of the TR-PPDR signal to change in $\tau(>0)$ exhibited in Fig. 2(b) indicates that the modulation of emission in the probe reflection direction by coherent and incoherent excitons induced by the pump pulse is not strongly distinguishable. In Fig. 3(b), the TR-PPDR signal at small $I_{1}$ shows an initial rise before decaying for larger $t$. As $I_{1}$ is increased, the rising part is 
not seen, the signal decaying continuously with $t$. If the decay of the coherent emission in the reflected probe pulse direction were exponential in $t$, and if the pump modulation only enhances the exponential decay rate, the TR-PPDR signal should be given simply as a difference of two exponentially decaying terms which may show an initial rise and then a decay. We find that the data of Fig. 2 (a) $(\tau<0)$ can be fitted by the two-exponential form. However, it is difficult to fit the + ve delay data [Fig. 2(b)] this way.

To compare experiments with theory, coupled Maxwell-Bloch equations have to be solved $^{21,22}$ with appropriate boundary conditions and many body Coulomb effects to obtain the PPDR signal for layered semiconductor heterostructures as used in our experiments. However, the following simplified phenomenological approach may be useful in understanding the origin of the PPDR signal as a starting step. Here, the MQW structure is replaced by an effective single layer of exciton resonances. Neglecting the effects of multiple reflections within the barrier and well regions and within the cap layer and taking the same background refractive index for the barriers and the QWs as a first approximation, the reflectivity $R$ for the model sample structure (Fig. 1) can be written as

$$
R \propto\left|r_{0} \mathcal{E}_{2}+\mathcal{E}_{Q W} e^{i \phi}\right|^{2}
$$

where only terms linear in the reflection coefficient $r_{0}(<1)$ of the cap layer on top of the QWs are retained. Here $\mathcal{E}_{2}$ is the amplitude of the probe electric field incident on the MQW and $\mathcal{E}_{Q W}$ is that of the field emitted from the MQW layer. The phase factor $\phi=2 d n \Omega / c$ arises from propagation delay for the probe pulse in the region between the vacuum-sample and top layer-MQW interfaces, $n$ is the refractive index of the cap layer, $c$ is the speed of light in vacuum and $\Omega$ is the circular frequency of the incident light. The PPDR signal $\Delta R$ is defined as $R_{\text {on }}-R_{\text {off }}$ where $R_{\text {on }}\left(R_{\text {off }}\right)$ is obtained from Eq. (11) when the QWs are excited (not excited) by the pump pulse. In calculating $R_{\text {on }}$ we assume that the pump pulse causes modulation $\Delta \mathcal{E}_{Q W}$ in $\mathcal{E}_{Q W}$ but $r_{0}$ remains unaffected.

For a thin sample with negligible depletion of incident pulse intensities, and using slowly varying envelope approximation in time, it can be shown ${ }^{21,23}$ that $\mathcal{E}_{Q W}$ in absence of pump pulses is proportional to $i \mathcal{P}_{2}^{(1)}$ and the modulation $\Delta \mathcal{E}_{Q W}$ caused by the pump pulse is proportional to $i \mathcal{P}^{N L}$, where $\mathcal{P}_{2}^{(1)}$ and $\mathcal{P}^{N L}$ are the amplitudes of the linear polarization induced by the probe pulse and nonlinear polarization induced by the pump and probe pulses, respectively. The lowest order term in $\mathcal{P}^{N L}$ is the third order polarization $\mathcal{P}^{(3)}$ which has products of the three amplitudes $\mathcal{E}_{2}, \mathcal{E}_{1}$ and $\mathcal{E}_{1}^{*}$ in the present case $\left(\mathcal{E}_{1}\right.$ is the amplitude of the pump electric field incident on the MQW).

Keeping only the lowest order term in $\mathcal{P}^{N L}$, we get the TR-PPDR signal as

$$
\begin{aligned}
\Delta R(t, \tau) \propto & -\operatorname{Im}\left[\mathcal{E}_{2}^{*}(t) \mathcal{P}^{(3)}(t, \tau) e^{i \phi}\right] \\
& +C \operatorname{Re}\left[\mathcal{P}_{2}^{*(1)}(t) \mathcal{P}^{(3)}(t, \tau)\right],
\end{aligned}
$$

where $t$ is the real time and $C=2 \pi \Omega n / c r_{0}$. Similarly, the SR-PPDR signal is given by

$$
\begin{aligned}
\Delta R(\omega, \tau) \propto & -\operatorname{Im}\left[\mathcal{E}_{2}^{*}(\omega) \mathcal{P}^{(3)}(\omega, \tau) e^{i \phi}\right] \\
& +C \operatorname{Re}\left[\mathcal{P}_{2}^{*(1)}(\omega) \mathcal{P}^{(3)}(\omega, \tau)\right]
\end{aligned}
$$

where $\mathcal{E}_{2}(\omega), \mathcal{P}_{2}^{(1)}(\omega)$, and $\mathcal{P}^{(3)}(\omega)$ are the Fourier transforms of $\mathcal{E}_{2}(t), \mathcal{P}_{2}^{(1)}(t)$, and $\mathcal{P}^{(3)}(t)$ respectively. Eqs. (2) and (3) apply also for the PPDT case when the phase term $\phi$ is set to zero and $r_{0}$ in $C$ is replaced by unity. The literature reports which are mostly concerned with SR-PPD measurements usually ignore 8.14 .15 the second term in Eq. (3). In our investigation of the SR-PPDR case ${ }^{11}$, it was found that the second term is indeed smaller than the first but is not negligible. We wish to emphasize here that unlike the SR-PPD case, the second term is very crucial for the TR-PPDR (and TR-PPDT) measurements. In fact, within the above model, it is easy to show that the TR-PPDR measurements can not be explained without the second term in Eq. (2).

Before evaluating $\Delta R(t, \tau)$, we may examine Eq. (2) to understand qualitatively how the TR-PPDR signal arises. For this, we refer to the schematic pictures of Fig. 4 Let us assume that the probe and pump pulses are $\delta$-like in time. Consider the -ve delay case. The probe pulse is taken to arrive on the sample at $t=0$ and the pump pulse later at $t=|\tau| \cdot \mathcal{P}^{(3)}(t, \tau)$ is nonzero (and decaying with $t$ ) only for $t \geq|\tau|$. In the first term of Eq. (2) $\mathcal{E}_{2}(t)$ is a $\delta$-like function at $t=0$. There is no overlap between $\mathcal{E}_{2}(t)$ and $\mathcal{P}^{(3)}(t, \tau)$ and hence no TR-PPDR signal is expected [Fig. 4 (upper panel)] from the first term. However in the second term, $\mathcal{P}_{2}^{(1)}(t)$ is nonzero (and decaying with $t$ ) for $t \geq 0$. If $|\tau|$ is less than the exciton dephasing time, then there is an overlap in $t$ between $\mathcal{P}_{2}^{(1)}(t)$ and $\mathcal{P}^{(3)}(t, \tau)$ for $t \geq|\tau|$ and we get nonzero TR-PPDR signal [Fig. 4 (upper panel)] due to the second term in Eq. (2). In the case of + ve delay, the pump pulse arrives on the sample at $t=-|\tau|$ and the probe pulse at $t=0$. $\mathcal{P}^{(3)}(t, \tau)$ is nonzero 


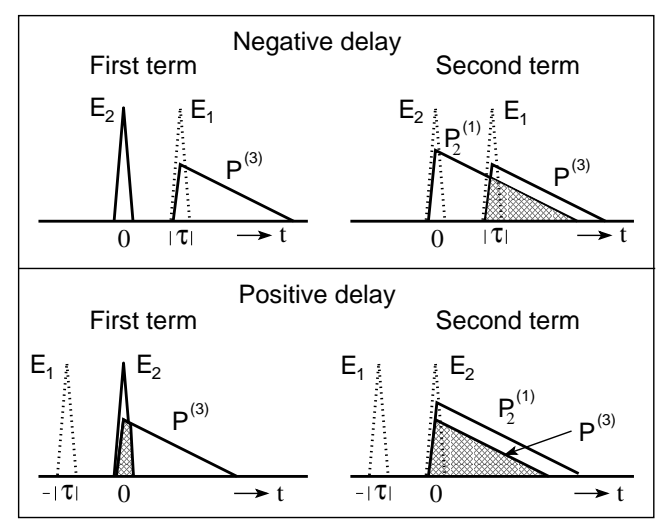

FIG. 4: Schematic representation of the origin of the TR-PPDR signal due to the two terms in Eq. (2) at -ve (upper panel) and + ve (lower panel) delay. The shaded regions indicate the time domain in which the TR-PPDR signal arises due to overlap of $\mathcal{P}^{(3)}(t)$ in $t$ with $\mathcal{E}_{2}(t)$ (first term) and $\mathcal{P}_{2}^{(1)}(t)$ (second term).

only for $t \geq 0$ and decays with $t$. There is an overlap between $\mathcal{E}_{2}(t)$ and $\mathcal{P}^{(3)}(t, \tau)$ at $t=0$ and between $\mathcal{P}_{2}^{(1)}(t)$ and $\mathcal{P}^{(3)}(t, \tau)$ for $t \geq 0$. Hence for + ve delay, the first term contributes only at $t=0$ and the second term contributes for $t \geq 0$ [Fig 4 (lower panel)]. In view of the above, observation of nonzero TR-PPDR signal (and in fact TR-PPDT signal as well) at $t>0$ for both + ve and - ve $\tau$ in Figs. 2 and 3 can not be explained if only the first term in Eq. (2) is considered.

Furthermore, it is obvious from the $t$ ordering of $\mathcal{E}_{2}(t)$ and $\mathcal{P}^{(3)}(t, \tau)$ that the time integral of the first term in Eq. (2) vanishes for $\tau<0$, giving no TI-PPDR signal. Thus, once again, the observed nonzero TI-PPDR signal for - ve $\tau$ in Fig. 1(c) can arise only from the $t$ integration of the second term in Eq. (2) (which does not vanish). Equivalence of TI- and SI-PPDR signal can be established by using Parseval's theorem for Eqs. (22) and (33). This immediately shows that the first term in Eq. (3) will lead to zero SI-PPDR signal, though it contributes to SR-PPDR measurements.

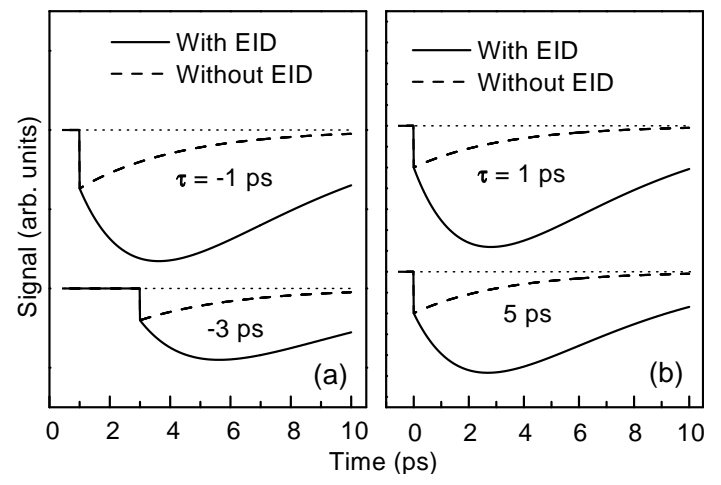

FIG. 5: Simulation of TR-PPDR signal using Eq. (2) calculated within optical Bloch equation for $\delta$-like electric fields for - ve (a) and + ve (b) delays, highlighting the role of excitation induced dephasing (EID). The signal is - ve and the curves for different $\tau$ are shifted along the vertical axis (linear scale), with the zero of the signal shown as dotted lines.

We now assess how far a calculation of $\mathcal{P}_{2}^{(1)}(t)$ and $\mathcal{P}^{(3)}(t, \tau)$ in Eq. (2) based on a perturbative solution of the coupled optical Bloch equations for a non-interacting two-level assembly up to third order in electric fields can reproduce the features shown by the experimental data. Many body effects like local fields (LF), excitation induced dephasing (EID) and resonance energy shift (ES) are included phenomenologically 11.17 .18 in this simplified approach. Although not done here, the coexcitation of hh and lh excitons can be easily included by modeling the excitonic assembly as a three-level system ${ }^{11,12}$. $\delta$-like pulses are assumed for computational ease although finite pulse widths can also be used. We calculate the time evolution of the PPDR signal $[\Delta R(t, \tau)]$ for both - ve and + ve $\tau$ (Fig. [5). Since the first term in Eq. (2) contributes only for +ve delay at $t=0$ in a time domain of the size of the pulse width, we display in Fig. 5 only the calculation of the second term in Eq. (2). We find that only EID contributes to $\Delta R(t, \tau)$ apart from the phase space filling related nonlinearity. The peak behavior seen in Fig. 5 is in fact a consequence of EID in this model. The LF and ES related terms lead the EID term by a phase of $\pi / 2$ and do not contribute to the 
TR-PPDR. (However, they do contribute to the SR-PPDR signal together with EID 11 .) In this model, the signal peak position in $t$ is essentially determined by an exciton density independent dephasing rate. The EID introduced in the Bloch equations does not lead to density dependent decay of $\mathcal{P}^{(3)}(t, \tau)$. This is consistent with the data of Fig. 3 Also, the model does lead to a delayed rise of the signal as observed in Fig. 22 Within this theory, the $t$ integration of the second term in Eq. (2) produces a nonzero signal for $\tau<0$, with the behavior $\sim \exp \left(2 \tau / T_{2}\right), T_{2}$ being the exciton dephasing time. This agrees with the data plotted in Fig. 1( $(\mathrm{c})$, giving $T_{2} \approx 4 \mathrm{ps}$, which is close to the value obtained from FWM measurements on this sample.

However, some features in the data are not explained by the simple theory. For example, the origin of the different rising behavior in Figs. 2 and 3 for - ve and +ve $\tau$ is not clear. Furthermore, although the TR-PPDR signal decay appears to be not very sensitive to increase in the pump intensity (Fig. 3i), the rise is. This is not accounted for in the above theory. Introduction of a phenomenological dependence of the dephasing time for the probe induced exciton polarization on pump generated excitation density may be considered, in analogy with the approach followed in the literature for $\mathrm{FWM}^{18}$. This will indeed cause a faster rise of the TR-PPDR signal in $t$ with increased pump intensity $\left(I_{1}\right)$. However, this will also lead to a faster decay in $t$ of the signal with increasing $I_{1}$, which is not evident in Fig. 3 A quantitative understanding of the $t$ and $I_{1}$ dependence exhibited by the data of Figs. 2 and 3 may be possible when an appropriate theory of the nonlinear optical interactions of the excitonic polarization, populations and propagating electric fields, that also takes into account the MQW nature of the sample and higher order Coulomb interactions, is applied. This is beyond the scope of this paper.

Among the important effects that may play some role in the TR-PPDR measurements is the following. It has been suggested 21.24 .25 that radiative coupling of excitations in individual QWs in a MQW structure may give rise to a few bright polaritonic modes which reradiate in the reflection direction more efficiently than other modes. One consequence of this theory is that the tail of the emission from the linear polarization may show a finite rise time and beats due to polaritonic interference. Indeed, it was deduced recently ${ }^{24}$ from measurements of time integrated reflectivity of a pair of phase locked pulses using an interferometric technique that the excitonic reflection in GaAs MQWs shows a finite rise time at low excitation intensities.

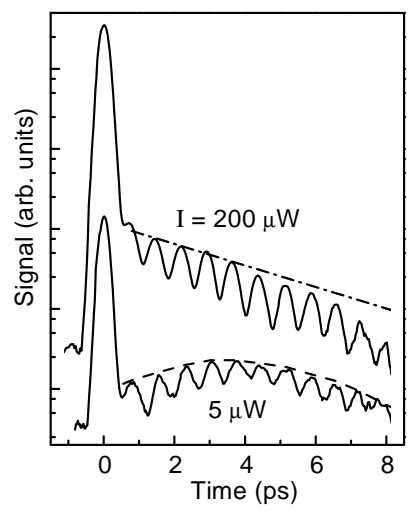

FIG. 6: Semilogarithmic plot showing the time profile of the coherent emission along the probe reflection direction (no pump pulse) for two average intensities $(I=200$ and $5 \mu \mathrm{W})$ of the incident pulse. Data at two $I$ are shifted vertically. The dash-dotted line is an exponential fit, the dashed curve is drawn to guide the eye.

To investigate this aspect in our case, the temporal profile of the reflected probe pulse is measured in absence of a pump pulse (Fig. 6). The strong peak at $t=0$ is essentially due to reflection of the probe pulse at the vacuum-sample interface. The oscillations seen are quantum beats due to coherent excitation of hh and lh excitons. The exciton polarization emits coherently for several ps. The emission is shown for two values of the average intensity $I$ of the incident pulse, namely $I=200$ and $5 \mu \mathrm{W}$. A simple estimate gives an exciton density of $8 \times 10^{6} \mathrm{~cm}^{-2}$ per $\mu \mathrm{W}$ per QW. At low intensities, the emission shows a finite rise time, the signal peaking at about 3 ps. This behavior is consistent with the possibility that polaritonic interference has an important effect on the dynamics of excitonic polarization in MQWs and may influence TR-PPDR measurements. This feature is not seen for the high intensity case, possibly due to reduced photon induced coupling between the excitons in the individual QWs caused by exciton dephasing. However, even in this case, the decay of the signal shows a departure from a simple exponential behavior, as indicated in Fig. 6] We should mention that the $t$ dependence seen in Fig. [6] is different from the nonexponential decay that may be caused by exciton inhomogeneous broadening when it is comparable to or more than the homogeneous broadening.

To conclude, the TR-PPDR results presented here reveal new features related to the time dynamics of excitonic polarization in MQWs and the dependence of pump modulation effects on pump intensity, pump-probe relative polarization and delay. A nonlinear optical contribution arising from interference of excitonic linear and nonlinear 
polarization, which was not considered earlier in the literature, was shown to be essential for understanding the TR-PPDR measurements.

We are grateful to L. N. Pfeiffer and J. Shah for the MQW sample.

* Electronic address: bipulpal@tifr.res.in

1 D. Weber, J. Feldmann, E. O. Göbel, T. Stroucken, A. Knorr, S. W. Koch, D. S. Citrin, and K. Köhler, J. Opt. Soc. Am. B 13, 1241 (1996).

2 S. Haacke, R. A. Taylor, R. Zimmermann, I. Bar-Joseph, and B. Deveaud, Phys. Rev. Lett. 78, 2228 (1997).

${ }^{3}$ G. R. Hayes, J. L. Staehli, U. Oesterle, B. Deveaud, R. T. Phillips, and C. Ciuti, Phys. Rev. Lett. 83, 2837 (1999).

${ }^{4}$ D. S. Kim, J. Shah, D. A. B. Miller, T. C. Damen, W. Schäfer, and L. Pfeiffer, Phys. Rev. B 48, 17902 (1993).

${ }^{5}$ O. Lyngnes, J. D. Berger, J. P. Prineas, S. Park, G. Khitrova, H. M. Gibbs, F. Jahnke, M. Kira, and S. W. Koch, Sloid State Commun. 104, 297 (1997).

${ }^{6}$ B. Fluegel, N. Peyghambarian, G. Olbright, M. Lindberg, S. W. Koch, M. Joffre, D. Hulin, A. Migus, and A. Antonetti, Phys. Rev. Lett. 59, 2588 (1987).

7 M. Joffre, D. Hulin, A. Migus, A. Antonetti, C. B. Guillaume, N. Peyghambarian, M. Lindberg, and S. W. Koch, Opt. Lett. 13, 276 (1988).

8 J. P. Sokoloff, M. Joffre, B. Fluegel, D. Hulin, M. Lindberg, S. W. Koch, A. Migus, A. Antonetti, and N. Peyghambarian Phys. Rev. B 38, 7615 (1988).

9 U. Neukirch, S. R. Bolton, L. J. Sham, and D. S. Chemla, Phys. Rev. B 61, R7835 (2000).

10 T. Guenther, C. Lienau, T. Elsaesser, M. Glanemann, V. M. Axt, T. Kuhn, S. Eshlaghi, and A. D. Wieck, Phys. Rev. Lett. 89, 057401 (2002).

11 Bipul Pal and A. S. Vengurlekar, (unpublished).

12 S. Schmitt-Rink, D. Bennhardt, V. Heuckeroth, P. Thomas, P. Haring, G. Maidorn, H. Bakker, K. Leo, D. S. Kim, J. Shah and K. Köhler, Phys. Rev. B 46, 10460 (1992).

13 J. Shah, Ultrafast Spectroscopy of Semiconductors and Semiconductor Nanostructures, $2^{\text {nd }}$ ed., (Springer, Berlin, 1999).

14 M. Lindberg and S. W. Koch, Phys. Rev. B 38, 7607 (1988).

15 S. W. Koch, N. Peyghambarian, and M. Lindberg, J. Phys. C 21, 5229 (1988).

${ }^{16}$ K. Leo, M. Wegener, J. Shah, D. S. Chemla, E. O. Göbel, T. C. Damen, S. Schmitt-Rink and W. Schäfer, Phys. Rev. Lett. 65, $1340(1990)$.

17 M. Wegener, D. S. Chemla, S. Schmitt-Rink, W. Schäfer, Phys. Rev. A 42, 5675 (1990).

18 H. Wang, K. B. Ferrio, D. G. Steel, P. R. Berman, Y. Z. Hu, R. Binder and S. W. Koch, Phys. Rev. A 49, R1551 (1994).

19 E. J. Mayer, G. O. Smith, V. Heuckeroth, J. Kuhl, K. Bott, A. Schulze, T. Meier, D. Bennhardt, S. W. Koch, P. Thomas, R. Hey, and K. Ploog, Phys. Rev. B 50, 14730 (1994).

20 C. Sieh, T. Meier, A. Knorr, F. Jahnke, P. Thomas, and S. W. Koch, Eur. Phys. J. B 11, 407 (1999).

21 T. Stroucken, A. Knorr, P. Thomas, and S. W. Koch, Phys. Rev. B 53, 2026 (1996).

${ }^{22}$ F. Jahnke, M. Kira, and S. W. Koch, Z. Phys. B 104, 559 (1997).

23 N. Bloembergen and P. S. Pershan, Phys. Rev. 128, 606 (1962).

24 J. J. Baumberg, A. P. Heberle, A. V. Kavokin, M. R. Vladimirova, and K. Köhler, Phys. Rev. Lett. 80, 3567 (1998).

25 J. P. Prineas, C. Ell, E. S. Lee, G. Khitrova, H. M. Gibbs, and S. W. Koch, Phys. Rev. B 61, 13863 (2000). 